\title{
Transmission parameters estimated for Salmonella typhimurium in swine using susceptible-infectious-resistant models and a Bayesian approach
}

Carla Correia-Gomes ${ }^{1,7^{*}}$, Theodoros Economou², Trevor Bailey², Pavel Brazdi ${ }^{3,4}$, Lis Alban ${ }^{5}$ and João Niza-Ribeiro ${ }^{1,6}$

\begin{abstract}
Background: Transmission models can aid understanding of disease dynamics and are useful in testing the efficiency of control measures. The aim of this study was to formulate an appropriate stochastic Susceptible-Infectious-Resistant/ Carrier (SIR) model for Salmonella Typhimurium in pigs and thus estimate the transmission parameters between states.

Results: The transmission parameters were estimated using data from a longitudinal study of three Danish farrow-to-finish pig herds known to be infected. A Bayesian model framework was proposed, which comprised Binomial components for the transition from susceptible to infectious and from infectious to carrier; and a Poisson component for carrier to infectious. Cohort random effects were incorporated into these models to allow for unobserved cohort-specific variables as well as unobserved sources of transmission, thus enabling a more realistic estimation of the transmission parameters. In the case of the transition from susceptible to infectious, the cohort random effects were also time varying. The number of infectious pigs not detected by the parallel testing was treated as unknown, and the probability of non-detection was estimated using information about the sensitivity and specificity of the bacteriological and serological tests. The estimate of the transmission rate from susceptible to infectious was $0.33[0.06,1.52]$, from infectious to carrier was $0.18[0.14,0.23]$ and from carrier to infectious was $0.01[0.0001,0.04]$. The estimate for the basic reproduction ration $\left(R_{0}\right)$ was $1.91[0.78,5.24]$. The probability of non-detection was estimated to be 0.18 [0.12, 0.25].

Conclusions: The proposed framework for stochastic SIR models was successfully implemented to estimate transmission rate parameters for Salmonella Typhimurium in swine field data. $R_{0}$ was 1.91 , implying that there was dissemination of the infection within pigs of the same cohort. There was significant temporal-cohort variability, especially at the susceptible to infectious stage. The model adequately fitted the data, allowing for both observed and unobserved sources of uncertainty (cohort effects, diagnostic test sensitivity), so leading to more reliable estimates of transmission parameters.
\end{abstract}

Keywords: Salmonella typhimurium, Transmission parameters, Bayesian approach

\footnotetext{
* Correspondence: carla.gomes@sruc.ac.uk

${ }^{1}$ ICBAS-UP - Department of Population Studies, Instituto de Ciências Biomédicas

Abel Salazar - Universidade do Porto, Rua de Jorge Viterbo Ferreira, n²28,

4050-313 Porto, Portugal

${ }^{7}$ ERU-SRUC - Epidemiology Research Unit, Future Farming Systems, SRUC,

Drummondhill, Inverness, IV2 4JZ, UK

Full list of author information is available at the end of the article
}

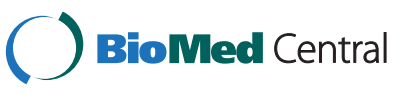

(c) 2014 Correia-Gomes et al.; licensee BioMed Central Ltd. This is an Open Access article distributed under the terms of the Creative Commons Attribution License (http://creativecommons.org/licenses/by/2.0), which permits unrestricted use, distribution, and reproduction in any medium, provided the original work is properly credited. The Creative Commons Public Domain Dedication waiver (http://creativecommons.org/publicdomain/zero/1.0/) applies to the data made available in this article, unless otherwise stated. 


\section{Background}

Salmonella Typhimurium is one of the major foodborne pathogens currently causing disease in humans [1] and it is often related to consumption of pork products. Given its relevance to consumer food safety, Salmonella spp. control was considered necessary by the European food safety policy makers under the EC Regulation 2160/2003. In the near future, it is possible that a mandatory target reduction will be put in place in the European Union, regarding the Salmonella prevalence for pigs.

In practice, however, the control of this agent has proved to be difficult and expensive at the farm level [2]. Consequently, evaluating the efficiency of control strategies relating to this agent has become an important issue, as stated in recent reports [3]. Modelling the dynamics of Salmonella Typhimurium in pigs is important in evaluating alternative control strategies. The basic reproduction ratio $\left(R_{0}\right)$ expresses the number of secondary cases to which a primary case gives rise during the infectious period and provides valuable information for simulation models. If $R_{0}$ is less than unity then the disease is receding, but when it is higher than unity the disease is spreading.

Susceptible - Infectious - Resistant (SIR) models are attractive tools that aid understanding of disease dynamics. The SIR model formulates the changes of individuals in the population between different disease states in terms of a system of ordinary differential equations (ODE), known as the Kermack-McKendrick ODE model [4]. The variables in the system are given by the three components groups: susceptible (S), infectious (I) and carriers (R). SIR models include a mathematical specification of the movement into and out of the three components. The key parameter in each of these mathematical specifications is the transition rate: from $S$ to I $(\beta)$, from I to $R(\alpha)$ and from $R$ to $I(v)$. If such modelling is to be helpful in infectious disease control, it is critical to have the best possible estimates of these rates $(\beta, \alpha$ and $v)$, as all three are important for modelling the spread of infection. Transmission data, generated under controlled conditions (i.e. experimental studies or controlled field studies) are necessary to estimate the transition rates as accurately as possible.

In most cases Salmonella Typhimurium causes subclinical infection in swine with no apparent symptoms of disease. This makes it difficult to assess the infection status of individual pigs in an infected population without testing each animal several times. One of the difficulties in obtaining accurate estimates for $\beta$ in Salmonella Typhimurium studies stems from the fact that the currently available bacteriological and serological tests used to assign the infection status are imperfect, introducing uncertainty when trying to classify each animal. Yet another source of uncertainty comes from the fact that pigs, once infected, shed the agent intermittently.

In the literature, it is conventional to use generalised linear models (GLMs) to describe the counts of animals moving between states (e.g. from $\mathrm{S}$ to I) using either Poisson [5-8] or Binomial distributions [4,9]. GLMs can be used to estimate all three transmission parameters although they lack flexibility, for example in capturing the effect of sensitivity and specificity of the diagnostic test used. GLMs also lack the flexibility to allow for unobserved effects of variables not recorded in the data, but which influence the outcomes. The Bayesian framework proposed in this paper is flexible enough to incorporate such effects and also quantify the uncertainty due to imperfect diagnostic tests.

Following cohorts of animals in order to determine the dynamics of $S$. Typhimurium in susceptible populations is a very expensive procedure and only a few such studies exist. In this paper, we use data from a previous observational study designed and performed by Kranker et al. [10].

A Bayesian modelling framework was proposed and used to estimate transmission parameters (transition rate from $S$ to $I$, transition rate from $I$ to $R$ and transition rate from $\mathrm{R}$ to $\mathrm{I}$ ) for Salmonella Typhimurium in pig herds, using the longitudinal data from Kranker et al. [10]. The sensitivity and specificity of the tests used to classify the animals in the Kranker study were allowed for in the statistical model, which also incorporated random effects to allow for cohort heterogeneity.

\section{Methods}

Study herds, sampling, bacteriology and ELISA test

The data used have been previously described by Kranker et al. [10] and originate from three Danish pig herds known to be infected with Salmonella Typhimurium. The herds had moderate to high levels of Salmonella Typhimurium and therefore the within-herd prevalence was $40 \%$ or higher. These measures of prevalence were based on meat-juice samples collected over three months, evaluated by use of an optical density (OD) cut-off of $20 \%$. Two of the farms, with 650 and 440 sows, respectively, were twosite operations; the remaining farm was a three-site operation with 300 sows. The three herds were self-supplying. In each herd, 10 litters were randomly selected, and in each litter, the ears of six randomly selected piglets were tagged. To account for variations in Salmonella shedding over time, litters from each herd were divided into two groups of five litters that were raised at approximately one-month intervals. Thus, on each farm there were two cohorts consisting of 30 pigs each, yielding a total of 180 piglets at the start of the study. All ear-tagged pigs from a given cohort were raised together for the entire observation period. The animals were followed longitudinally 
[10] and were first tested at the age of four weeks and thereafter at two to five week intervals until the age of slaughter. Slaughter age varied between cohorts but was on average around 25 weeks. Cohorts were tested either six to seven times in total. On each testing occasion, sera and faeces from the animals were collected and tested for the presence of Salmonella spp.. At the age of four weeks only faeces were collected, because maternal antibodies still present could give a false positive result. An animal was considered serologically positive, wherever the serological test revealed a result of OD\% $>20$, and bacteriologically positive if Salmonella was isolated from the faeces. The serological test used at this cut-off value is considered to have a sensitivity of $68 \%$ and to be $100 \%$ specific [11]. The bacteriological test is considered to be $100 \%$ specific and the sensitivity is around 30 to $55 \%$ [12]. These test characteristics were incorporated in the statistical model.

\section{Infection status of the pigs}

The testing time interval was different in each cohort, varying from two to five weeks. A homogenous dataset was derived by inferring the infection status of each pig, every two weeks. The time step of two weeks was chosen because on average it takes two weeks for an animal to test positive to serology after being infected. It was therefore assumed that an animal was infectious in the two weeks before being seropositive. The most likely infection status of each pig was determined for each two-week time step based on both faecal shedding and serology results from the sampling period closest to each time step. Each animal was categorized as susceptible (S), infectious (I) or carrier (R). An animal was considered susceptible if the agent was not present and the animal was at the risk of infection. An animal was considered infectious if it was shedding the agent and a potential source of infection to other animals. An animal that was infected with the agent but not shedding and therefore not able to infect other animals was considered a carrier. In the absence of reasonable sensitivity of the bacteriological culture method, serology offered an alternative and complementary way to assign the infection status of a pig and both methods were used in parallel to categorise the pigs' status.

Pigs were attributed status $\mathrm{S}$ when there was no presence of bacteria in the faecal samples and the OD\% was below 20. Status I was assigned from the date when a pig was found to be bacteriologically positive until it was no longer positive by this testing method. Additionally, pigs were assigned to status I based on seroconversion. The beginning of the infectious period was set to two weeks prior to the recorded date of seroconversion $[13,14]$ and the duration was set to four weeks, assuming that a pig would shed Salmonella spp. within an average of four weeks. This average period was based on experimental data regarding duration of the shedding period $[13,15]$. Thus, information was used from both tests in parallel for pig classification. Finally, status I was followed by status $R$ and the pigs could return to status I if they were found to be culture positive later on during the study period. It was assumed that no pig would return to the susceptible status after being infected, because of the relative short life span of finisher pigs (after infection it takes around 112 days to clear the agent from the organs [14], and post-weaned pigs are generally slaughtered before this time). The following describes a particular example of how the classification was performed: if a pig was shedding at a specific testing time, it was considered infectious in the nearest bi-weekly time step, until it became bacteriologically negative, after which it was considered a carrier (in the nearest bi-weekly time step). If a pig was serologically positive, in the presence of a negative culture, it was considered infected and therefore classified as infectious for at least four weeks, from the nearest bi-weekly time step prior the testing time. If an animal was bacteriologically and serologically negative, it was considered susceptible. Given that testing of piglets was restricted to bacteriology (which has low sensitivity) at the beginning of the follow-up period, some piglets infected by the sow could have been erroneously classified as susceptible. For this reason, the analysis in each cohort started at the time infected animals were first detected (by either serology or bacteriology).

\section{Estimation of the transmission parameters}

Conventionally, transmission parameters of infectious disease, including Salmonella spp., in swine herds [14,16-21] are estimated using regression models. These are often based on data describing the prevalence of that disease in the country or region to which the particular study refers. As suggested in some studies [5,22,23], we first applied a stochastic SIR models in the form of Generalised Linear Models (GLMs) in order to estimate the three transmission parameters. However, preliminary results (not reported here) suggested the presence of overdispersion in the GLMs, hinting towards unobserved sources of variation in the data such as cohort heterogeneity. Instead, we here report a framework for stochastic SIR models which i) extends the current GLM framework by including random effects, ii) is implemented using a Bayesian approach thus allowing incorporation of prior information (such as the sensitivity of Salmonella tests), iii) explicitly estimates the probability of not detecting infectious animals due to test sensitivity and iv) incorporates all sources of uncertainty/variation thus obtaining more realistic estimates of transmission parameters. As suggested by some authors [5], the inclusion of 
random effects automatically accounts for overdispersion by inflating the variance of the response variable while at the same time allowing for cohort heterogeneity.

Stochastic SIR models (and other variants such as SI or SIS models) are well-established in animal disease literature [4,19-21]. The benefit of using a stochastic SIR model is that transmission parameters can be estimated using statistical modelling; here the conventional stochastic SIR model was extended by explicitly allowing for cohort variation and unobserved temporal effects. The three components of the stochastic SIR model are described in detail below.

\section{Transition from susceptible to infectious}

It was assumed that pigs become infected by "infectious contacts" defined as: either contact with other infected animals, or contact with their environment (rodents, contaminated muck or feed). The rate at which a given animal has infectious contacts was assumed i) to be constant in time and ii) proportional to the density of infectious animals [19], with a constant of proportionality $\beta$, i.e. the transmission rate parameter. In other words, the infectious contacts per animal happen randomly in time so that their occurrence can be described by a Poisson process. More precisely, the number of infectious contacts per animal in a period $\Delta \mathrm{t}$ is Poisson distributed with mean $\lambda=\beta(I / N) \Delta t$, where I is the number of infectious animals and $\mathrm{N}$ is the total number of animals, at the beginning of $\Delta t$. As such, the probability of no infectious contacts per animal in $\Delta t$ is $\exp (-\beta(\mathrm{I} / \mathrm{N}) \Delta \mathrm{t})$, implying that the probability of infection in $\Delta t$ is $p=1-\exp$ $(-\beta(\mathrm{I} / \mathrm{N}) \Delta \mathrm{t})$. This means that the number of new cases $C$ at the end of $\Delta t$ is Binomial with parameters $\mathrm{S}$ and $\mathrm{p}$ so that the mean of $\mathrm{C}$ is $\mathrm{S}^{*} \mathrm{p}$.

Here, the current established methodology was extended to allow for the fact that i) $\lambda$ may vary in time due to exogenous factors and ii) $\lambda$ may vary across cohorts due to unobserved cohort effects. A random (scaling) effect exp $\left(r_{j t}\right)$ was included, for the $j^{\text {th }}$ cohort at time $t$, to get $\lambda_{j t}=\beta$ $(\mathrm{I} / \mathrm{N}) \exp \left(\mathrm{r}_{\mathrm{jt}}\right) \Delta \mathrm{t}$ as the mean number of infectious contacts of a random animal, in herd $j$ at time $t$. Note that $\Delta t$ denotes the length of a time interval whereas $t$ refers to actual time. On average, exp $\left(\mathrm{r}_{\mathrm{jt}}\right)$ was assumed to be equal to one, so that across all cohorts and time, the average transmission rate parameter is still $\beta$. By doing this, variation due to cohort or unknown temporal effects was explicitly modelled, which would otherwise contribute to the uncertainty in estimating $\beta$.

All time intervals in the data are equal to two weeks so for clarity, $\Delta t=1$ was set so that one time step $\Delta t$ corresponds to two weeks. This does not qualitatively affect the estimation of the transmission parameters. Because of the nature of the data, time $t$ is now defined in discrete consecutive (bi-weekly) time steps.
The model may be formulated as follows:

$$
\begin{aligned}
& \mathrm{C}_{\mathrm{jt}} \sim \operatorname{Binomial}\left(\mathrm{S}_{\mathrm{jt}}, \mathrm{p}_{\mathrm{jt}}\right) \\
& \mathrm{p}_{\mathrm{jt}}=1-\exp \left\{-\beta\left(\mathrm{I}_{\mathrm{jt}-1} / \mathrm{N}_{\mathrm{jt}-1}\right) \exp \left(\mathrm{r}_{1 \mathrm{jt}}\right)\right\} \\
& \operatorname{clog} \log \left(\mathrm{p}_{\mathrm{jt}}\right)=\log (\beta)+\log \left(\mathrm{I}_{\mathrm{jt}-1}\right)-\log \left(\mathrm{N}_{\mathrm{jt}-1}\right)+\mathrm{r}_{1 \mathrm{jt}}
\end{aligned}
$$

where:

- $\mathrm{C}_{j t}$ denotes the number of new infectious animals in cohort (j) at the end of the time step $(\mathrm{t})$,.

$-\mathrm{S}_{j-1}$ is the number of susceptible animals in cohort (j) at the end of the time step $(t-1)$,

- $\mathrm{p}_{\mathrm{jt}}$ is the probability of a susceptible animal in cohort (j) at the end of time step $(t-1)$ becoming infectious by the end of time step $(\mathrm{t})$,

- cloglog is the complementary log-log transformation,

- $\beta$ is the transmission rate parameter for the transition from susceptible to infectious,

- $\mathrm{I}_{j t-1}$ is the number of infectious animals in cohort $(\mathrm{j})$ at the end of the time step ( $t-1)$,

- $\mathrm{N}_{j t-1}$ is the total number of animals in cohort $(\mathrm{j})$ at the end of the time step ( $\mathrm{t}-1)$, and.

- $\mathrm{r}_{1 j t}$ is a cohort time-dependent random effect (which is zero on average).

Note that, at the beginning of the study, pigs were considered to be either in the S or I status depending on the test results. When there was no infectious pig present at the end of the previous time step, i.e. $I_{j t-1}=0$, the probability of becoming infectious was modelled as:

Cloglog $\left(p_{j t}\right)=\log (\beta)+r_{1 j t}$. This is because even if there are no infectious pigs present, animals can still be infected (e.g., contaminated environment, feed, water, etc.). In this formulation, $\beta$ is seen as the underlying rate of transition for a random pig in an average cohort with no infectious animals, while $r_{1 j t}$ allows for unobserved cohort-time effects in the data e.g., anthropogenic influence, rodents etc. Note that homogeneous mixing of the pigs in each cohort (i.e. all pigs could come into contact with each other) was assumed, due to the small size of the cohorts.

In using the number of infectious pigs $\mathrm{I}_{\mathrm{j} t}$, in each cohort at the end of time step $t$, it was necessary to account for the sensitivity of both the serological and bacteriological test. Since the specificity in both tests is considered to be $100 \%$, the parallel specificity is 1 . This implies that $I_{j t}=I_{o b s}{ }_{j t}+I_{n o b}$, where Iobs $_{j t}$ is the observed value and Inob $_{j t}$ is the number of infectious animals not detected (false negative pigs). In other words, $\operatorname{Iobs}_{\mathrm{jt}}$ is a lower bound on the actual $\mathrm{I}_{\mathrm{jt}}$. The unobserved variable Inob $_{j t}$ may be incorporated (and thus estimated) in the stochastic model and here it was assumed that it has a $\mathrm{Bi}$ nomial distribution with parameters $\mathrm{N}_{\mathrm{jt}}$ and $\mathrm{pND}$ where 
pND is the probability of not detecting infectious animals. This probability, pND, is of course dependent on the sensitivity probabilities of each test, which were assumed to be independent. Inob ${ }_{j t}$ was modelled as follows:

$$
\begin{aligned}
& \mathrm{I}_{\mathrm{jt}}=\mathrm{Iobs}_{\mathrm{jt}}+\mathrm{Inob}_{\mathrm{jt}} \\
& \text { Inob }_{\mathrm{jt}} \sim \operatorname{Binomial}\left(\mathrm{N}_{\mathrm{jt}}, \mathrm{pND}\right) \\
& \mathrm{pND}=(1-\operatorname{Sen} \mathrm{C})^{*}(1-\mathrm{SenE})
\end{aligned}
$$

where:

- SenC is the sensitivity probability of microbiological culture, and.

- SenE is the sensitivity probability of the ELISA test.

Treating Inob $\mathrm{j}_{\mathrm{jt}}$ as an unobserved random variable allows formal quantification of the uncertainty in the data due to test sensitivity and constitutes one of the novelties of the proposed model. The Bayesian framework (see section 5 later on) used to estimate the stochastic SIR model can easily incorporate the estimation of Inob $b_{j t}$ given prior information on SenC and SenE.

\section{Transition from infectious (I) to resistant (R)}

The rate $\alpha$ at which a random infectious animal in a given cohort becomes a carrier was assumed to be constant in time. As such, the length of time $\tau$ until an infectious animal becomes carrier can be modelled by an exponential distribution with rate parameter $\alpha$. So, given that the animal is infectious at the start of time interval $\Delta t$, the probability $\mathrm{pR}$ of becoming carrier is $\mathrm{pR}=\mathrm{Pr}$ $(\tau \leq \Delta t)=1-\exp (-\alpha \Delta t)$ since $\tau$ is exponentially distributed (recall that $\Delta t=1$ was set for conciseness). As before, a random cohort effect $r_{2 j}$ was added to allow for cohort heterogeneity in the data, to obtain $\mathrm{pR}_{\mathrm{j}}=1-\exp (-\alpha \exp$ $(\mathrm{r} 2 \mathrm{j})$ ). The number of new carrier animals $\mathrm{Rnew}_{\mathrm{jt}}$ at the end of time step $t$, is thus Binomial with parameters $I_{j t}$ and $\mathrm{pR}_{\mathrm{j}}$. Note that a single parameter $\alpha$ was utilised, describing the rate at which a random infectious animal in an average cohort, becomes carrier, however, cohort variability (not all cohorts are average) was allowed for through $r_{2 j}$, which in turn reduces uncertainty in estimating $\alpha$. The I to $\mathrm{R}$ transition was modelled as follows:

$$
\begin{aligned}
& \operatorname{Rnew}_{\mathrm{jt}} \sim \operatorname{Binomial}\left(\mathrm{I}_{\mathrm{jt}}, \mathrm{pR}_{\mathrm{j}}\right) \\
& \operatorname{clog} \log \left(\mathrm{pR} \mathrm{R}_{\mathrm{j}}\right)=\log (\alpha)+\mathrm{r}_{2 \mathrm{j}}
\end{aligned}
$$

\section{Transition from resistant to infectious}

For this compartment of the model, the rate of infectious contacts $v$ in a random carrier animal was assumed to be constant in time, where $v$ is the transmission rate parameter for the transition from carrier to infectious. With similar arguments as in the $\mathrm{S}$ to I compartment, the number of infectious contacts per animal in time period $\Delta t$ is Poisson distributed with mean $v \Delta t$. Since this transition was actually a rare event (only happening three times in the entire study), the Poisson distribution can be used, as it approximates the Binomial when its probability parameter is close to zero. So if in cohort j, there are $R_{\mathrm{jt}-1}$ carrier animals at the end of the previous time step, the number of transitions from $\mathrm{R}$ to $\mathrm{I}$ in time step $\mathrm{t}$ may be modelled as a Poisson variable with mean $\mu_{\mathrm{jt}}=v \mathrm{R}_{\mathrm{jt}-1} \exp \left(\mathrm{r}_{3 \mathrm{j}}\right)$. More explicitly:

$$
\begin{aligned}
& \operatorname{Inew}_{\mathrm{jt}} \sim \operatorname{Poisson}\left(\mu_{\mathrm{jt}}\right) \\
& \log \left(\mu_{\mathrm{jt}}\right)=\log (v)+\log \left(\mathrm{R}_{\mathrm{jt}-1}\right)+\mathrm{r}_{3 \mathrm{j}}
\end{aligned}
$$

where:

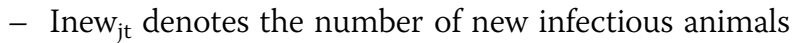
(that result from this transition) in cohort (j) at the end of the time step $(\mathrm{t})$,

- $\mu_{\mathrm{jt}}$ is the mean number of carrier animals that become infectious in the cohort $(\mathrm{j})$ during time step $(\mathrm{t})$,

$-v$ is the transmission rate parameter for the transition from carrier to infectious state,

- $\mathrm{R}_{\mathrm{jt}-1}$ is the number of carrier animals at the end of the time step $(t-1)$ in cohort $(j)$, and.

- $r_{3 j}$ is a cohort random effect that allows for cohort heterogeneity.

Note that $R_{j t-1}=0$ is possible, in which case $\log \left(R_{j t-1}\right)=0$ was set. The argument for doing this is that the transmission rate parameter $\nu$ may be defined as the limit of $\mu_{\mathrm{jt}} / \mathrm{R}_{\mathrm{jt}-}$ ${ }_{1}$ as $R_{\mathrm{jt}-1}$ goes to zero. As such, ignoring the random effect for a moment, $\mu_{\mathrm{jt}} / \mathrm{R}_{\mathrm{jt}-1}$ should tend to a constant (i.e. $v$ ) as $\mathrm{R}_{\mathrm{jt}-1}$ goes to zero rather than infinity. Note that in our data, $\mathrm{R}_{\mathrm{jt}-1}=0$ happened on $20 \%$ of occasions. In the hypothetical case that $R_{\mathrm{jt}-1}=0$ for the majority of time steps and cohorts, then this component of the model (i.e. the transition $\mathrm{R}$ to $\mathrm{I})$ becomes redundant as there will ultimately be almost no information with which to estimate the transition parameter.

\section{Cohort random effects}

As indicated above, random cohort effects were incorporated into each transition step to allow for i) cohort heterogeneity/variability in the data, ii) unobserved cohortspecific factors, iii) unobserved temporal effects in the $\mathrm{S}$ to I compartment. These effects were different for each transition under the assumption that any unobserved cohort factors affect each transition in a different way. For the transitions $\mathrm{S}$ to $\mathrm{I}$ and $\mathrm{R}$ to $\mathrm{I}$, these random effects also allow for factors which affect disease spread but which are not dependent on the animals themselves (such as contaminated environment, feed, water, etc.). 
For the transition $\mathrm{S}$ to $\mathrm{I}$, the cohort random effects were assumed to be time-varying and auto-correlated, and were modelled as:

$$
\begin{aligned}
& r_{1 j, t=1} \sim \operatorname{Normal}\left(0, \sigma_{1}^{2}\right) \\
& r_{1 j, t} \sim \operatorname{Normal}\left(r_{1 j, t-1}, \sigma_{1}^{2}\right)
\end{aligned}
$$

where the cohort random effect $\left(r_{1 j t}\right)$ for time step $t$ depends on the previous cohort random effect at time (t-1). With this cohort time-dependent random effect any unobserved dynamic behaviour in the spreading of infection within cohorts was captured, such as that due to infected mice.

For the transition I to $\mathrm{R}$ and $\mathrm{R}$ to $\mathrm{I}$, the random effects were modelled as:

$$
r_{k j} \sim \operatorname{Normal}\left(0, \sigma_{k}^{2}\right), k=2,3
$$

where:

- subscript $j$ denotes cohorts and.

- $\sigma_{\mathrm{k}}^{2}$ is the variance of the unobserved cohorts effects.

In a preliminary model building stage, a cohort timedependent random effect, $r_{2 j \mathrm{t}}$, was considered for the transition I to R; however the results showed no improvement to the model fit. Note that cohort time-dependent random effects were not considered for the Poisson model of the transition $\mathrm{R}$ to I. The transition only occurred three times in the entire study and it would be unreasonable to try to estimate unobserved temporal effects from this.

\section{Model implementation}

The overall SIR model described above was implemented in a Bayesian framework and fitted using Markov chain Monte Carlo (MCMC). In this framework, parameters are treated as random variables whose "prior" distribution expresses our uncertainty about their value before any data is observed. Prior distributions (priors) are combined with the observed data through Bayes theorem to produce the posterior distributions for each parameter (posteriors). The posteriors express the uncertainty about model parameters after data is observed and all statistical inference is based solely on the posteriors. MCMC is a numerical technique which produces samples of values that eventually converge (after a certain "burnin" number) to samples of values from the posterior (distribution) of each parameter.

There was no historical information with which to inform the prior distributions of $\log (\beta), \log (\alpha)$ and $\log (v)$, so Normal distributions with zero mean and a variance of 100 were used, reflecting prior ignorance while avoiding the use of improper prior distributions [24]. For the sensitivity probabilities of both serological and bacteriological tests, a Beta distribution was used as a prior. Previous information about the sensitivity of both tests $[11,12]$ was used to inform those Beta distributions: a mean of 0.49 for faecal culture and a mean of 0.68 for Danish mix ELISA were assumed, so SenC $\sim$ Beta $(48.5,50.5)$ and SenE $\sim$ Beta $(58.5,27.5)$ were specified. These priors have means 0.49 and 0.68 respectively, and variances that match the range of possible values dictated by the findings of $[11,12]$. Specificity was assumed to be $100 \%$ in both tests. The precision (i.e. the inverse of the variance) of the Normal distribution for each random effect was given a Gamma $(0.5,0.005)$ prior distribution (large mean and very large variance to indicate prior ignorance).

The complete SIR model was implemented in the open-source statistical software WinBUGS [25]. Exactly 100,000 posterior samples were collected after a 5,000 sample burn-in to ensure convergence to the posterior distribution [26]. Two MCMC runs were performed, with different initial values, to ensure convergence and mixing. The samples were thinned by only collecting one in 10 consecutive samples to eliminate autocorrelation in posterior samples (the R package "coda" [27] was used), so that in total we ended up with 20,000 samples. Convergence was assessed by inspection of trace-plots but also more formally using the Raftery and Lewis diagnostic, and the Gelman-Rubin R-hat diagnostic which should be sufficiently close to one if convergence has been achieved $[28,29]$. Mixing in the chains was assessed by comparing the Markov Chain (MC) error with the standard deviation, for each parameter. Ideally the MC error for each parameter should be less than $5 \%$ of the standard deviation [30] for good mixing.

Posterior predictive simulation was used for model checking as described by Gilks et al. [24]. This technique is effectively testing whether the observed data are extreme in relation to the posterior predictive distribution of the observations (i.e., the fitted model). The deviance was the measure adopted for comparison. The technique involves the calculation of a "p-value" which should not be extreme (close to 0 or 1 ) for good model fit.

\section{Calculations of the basic reproduction ratio $\left(\mathrm{R}_{0}\right)$}

Samples from the posterior distribution of $R_{O}$ were calculated from those of $\beta$ and $\alpha$ using the following formula [5]:

$$
R_{0}={ }^{\beta} / \alpha
$$

where $\beta$ is the transition rate from $S$ to $I$, and $\alpha$ is the transition rate from I to $\mathrm{R}$.

\section{Results}

\section{Transmission parameters}

Results, in terms of summary statistics from the posterior samples, are shown in Table 1 . Note that the posterior 
Table 1 Summary measures of the transmission parameters and random effects variances from the Salmonella transmission in pigs SIR model

\begin{tabular}{|c|c|c|c|c|c|c|c|c|}
\hline \multirow[t]{2}{*}{ Parameters } & \multirow[t]{2}{*}{ Mean } & \multirow[t]{2}{*}{ Standard deviation } & \multicolumn{5}{|c|}{ Quartiles } & \multirow[t]{2}{*}{ Rhat } \\
\hline & & & $2,5 \%$ & $25 \%$ & $50 \%$ & $75 \%$ & $97,5 \%$ & \\
\hline$\beta$ & 0.44 & 0.49 & 0.06 & 0.20 & 0.33 & 0.52 & 1.52 & 1.0021 \\
\hline$a$ & 0.18 & 0.02 & 0.14 & 0.17 & 0.18 & 0.20 & 0.23 & 1.0009 \\
\hline$v$ & 0.02 & 0.03 & 0.0001 & 0.006 & 0.01 & 0.02 & 0.04 & 1.0009 \\
\hline$\sigma_{1}^{2}$ & 3.00 & 1.80 & 0.80 & 1.76 & 2.60 & 3.77 & 7.59 & 1.0011 \\
\hline$\sigma_{2}^{2}$ & 0.02 & 0.04 & 0.002 & 0.005 & 0.01 & 0.02 & 0.096 & 1.0009 \\
\hline$\sigma_{3}^{2}$ & 6.64 & 38.82 & 0.003 & 0.06 & 0.08 & 3.85 & 44.44 & 1.0010 \\
\hline pND & 0.18 & 0.03 & 0.12 & 0.16 & 0.18 & 0.20 & 0.25 & \\
\hline $\mathrm{R}_{0}$ & 2.20 & 1.25 & 0.78 & 1.46 & 1.91 & 2.56 & 5.24 & \\
\hline
\end{tabular}

Legend: $\beta$ - transition rate from susceptible to infectious, $a$ - transition rate from infectious to carrier, $v$ - transition rate from carrier to infectious, $\sigma_{1}^{2}-$ variance of the random effects for the transition from susceptible to infectious, $\sigma_{2}^{2}$ - variance of the random effects for the transition from infectious to carrier, $\sigma_{3}^{2}$ - variance of the random effects for the transition from carrier to infectious, $p N D$ - probability of non-detection of infectious animals, $\mathrm{R}_{0}-$ basic reproduction ratio.

samples are MCMC samples from the posterior distribution of each model parameter and inference is based on those samples. A point estimate, the standard error and the $95 \%$ credible interval for a parameter are, for example, calculated as the sample mean, the sample standard deviation and the sample $2.5 \%$ and $97.5 \%$ quantiles of the posterior samples for that parameter.

The MCMC convergence was considered acceptable since the R-hat for all parameters (including random effects) was never above 1.01. The results of the model did not significantly differ when the parameters of the priors for the sensitivity tests were varied (increasing and decreasing them by $10 \%)$.

The posterior distribution for transition rate $\alpha$ (I to $R$ ) was symmetric, but for the transition rate $\beta$ (S to I) and $v$ ( $\mathrm{R}$ to $\mathrm{I}$ ), the posterior distributions were asymmetric (Figures 1, 2 and 3). As such, the posterior median was chosen to best summarise the value of these parameters. The median for the transition rate $\beta$ was 0.33 , for $\alpha$ it was 0.18 while for $v$ it was 0.01 (Table 1 ). The median of the variance of cohort random effects for the transitions I to $\mathrm{R}$ and $\mathrm{R}$ to I was close to zero, which implies that there was little significant variation between cohorts for these two transitions of the model. The median of the variance of the cohort-time dependent random effect for the transition $\mathrm{S}$ to I was 2.6 (95\% credible interval [0.80; $7.59]$ ), meaning that the cohort random effect is significant for this transition (Figure 4). The overall model fit was satisfactory with a "p-value" of 0.24 implying no significant difference between posterior predictive simulations (predictions from the model) and observed data.

\section{Basic reproductive ratio $\left(R_{0}\right)$}

Summary statistics of the posterior distribution of the $R_{O}$ parameter are shown in Table 1 . The posterior median of $R_{O}$ was 1.91 , with a $95 \%$ credible interval of 0.78 to
5.24. A density estimate of the posterior samples of $R_{O}$, which effectively describes the spread of Salmonella spp. in these three Danish pig herds known to be infected with Salmonella, is shown in Figure 5. For moderate to high within herd Salmonella prevalence, this $R_{O}$ distribution suggests that Salmonella Typhimurim can range from fading out scenarios to epidemic ones but most of the time the infection spread assumes an endemic form.

\section{Diagnostic test sensitivity (pND)}

Recall that this modelling framework includes the estimation of the probability of failing to detect infectious

\section{B}
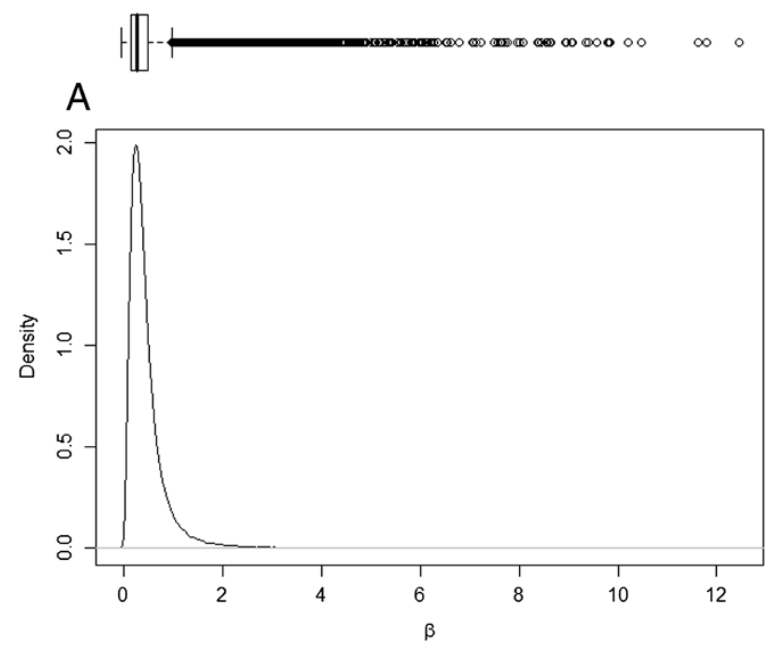

Figure 1 Posterior distribution of $\boldsymbol{\beta}$. Legend: $\mathbf{A})$ Plot of the posterior distribution for transmission parameter $\beta$, which describes the rate of spread of Salmonella Typhimurium from susceptible to infectious animals; B) Boxplot of the posterior samples used to produce the plot where the thick line in the box reflects the median. 


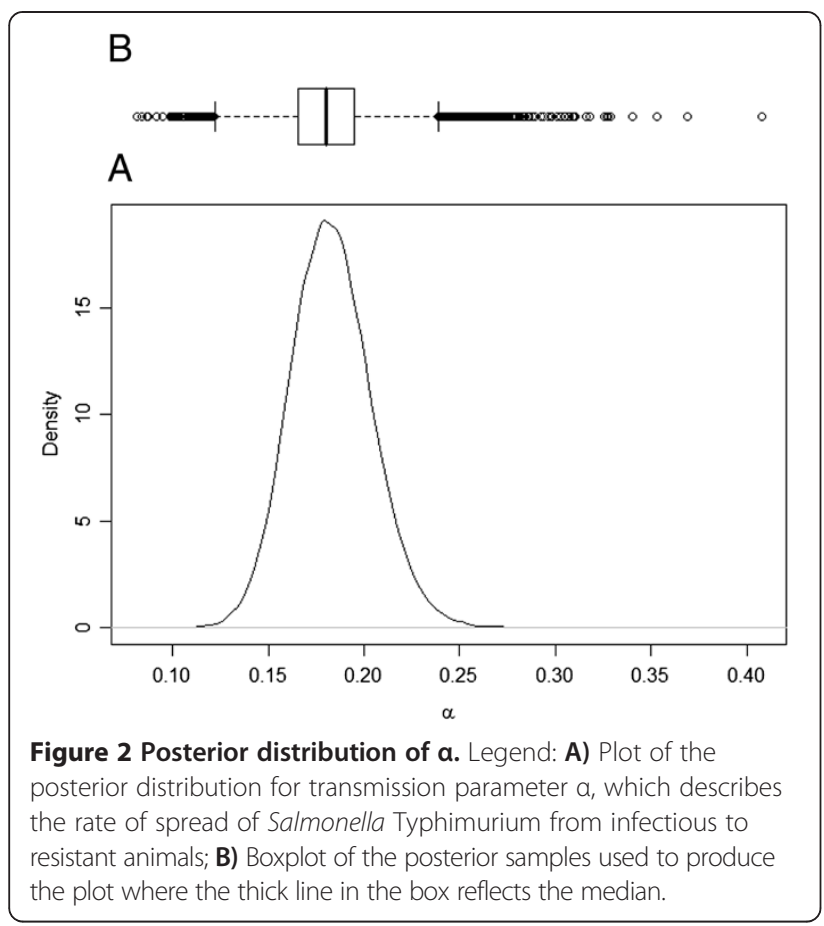

animals, pND, using both the data but also prior information about the tests sensitivity $[11,12]$. Figure 6 shows a density estimate plot of the posterior distribution of $\mathrm{pND}$.

\section{Discussion}

In this paper field data were used from a study conducted in three Danish pig herds which were known to be infected

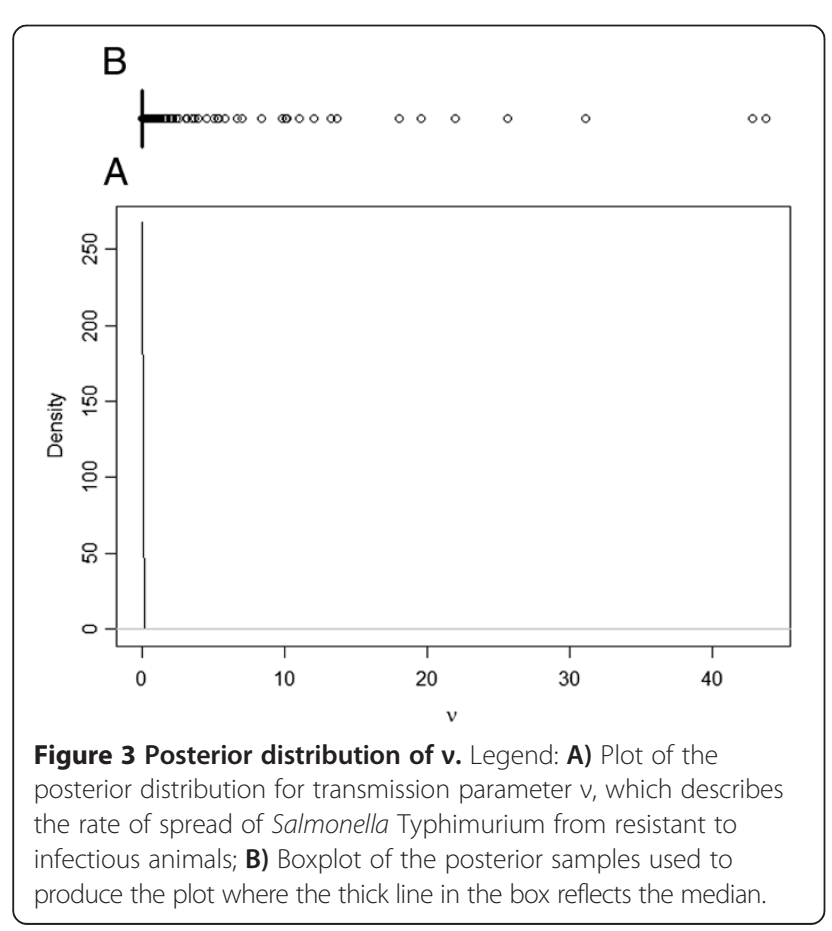

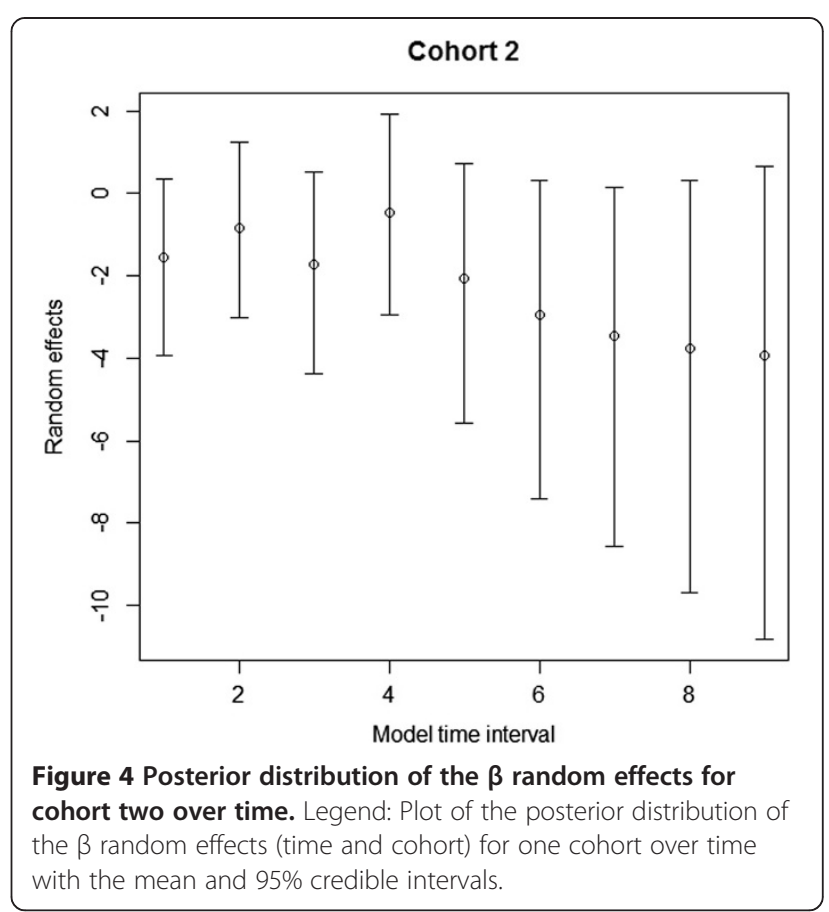

with Salmonella Typhimurium [10]. The data was considered sufficiently reliable to be used in estimating the transmission parameters of an SIR model describing the evolution of the disease and from that to infer $R_{O}$ for Salmonella Typhimurium in finisher pigs.

In comparing the parameters to those found in other studies (whether simulation- or observation-based studies)

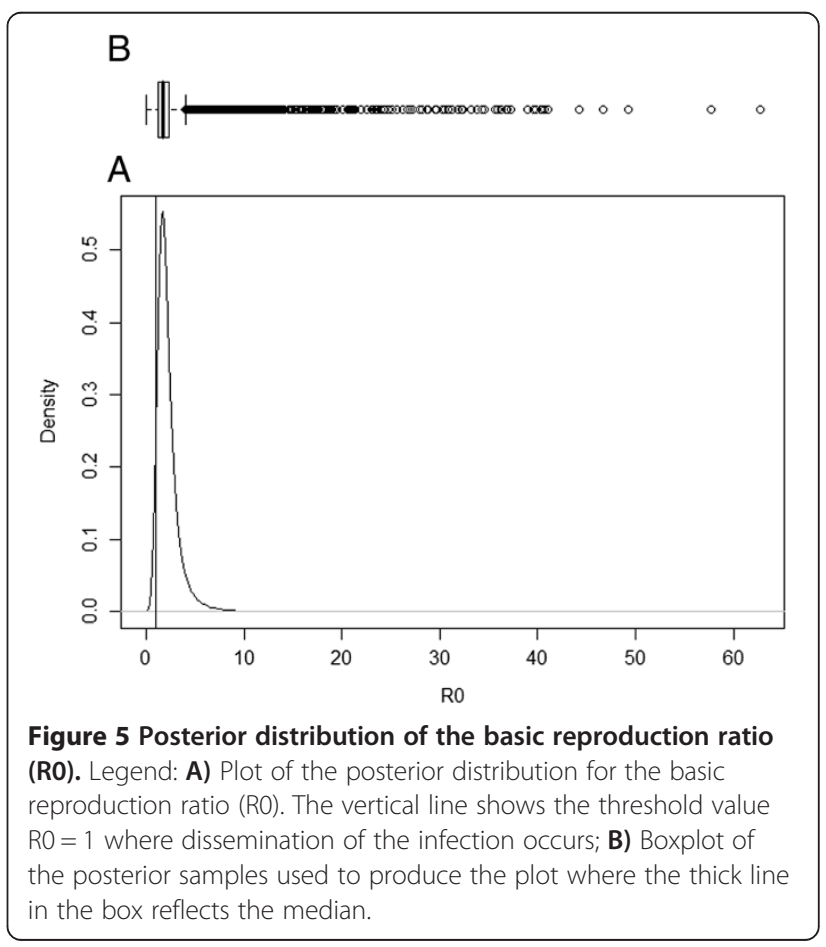




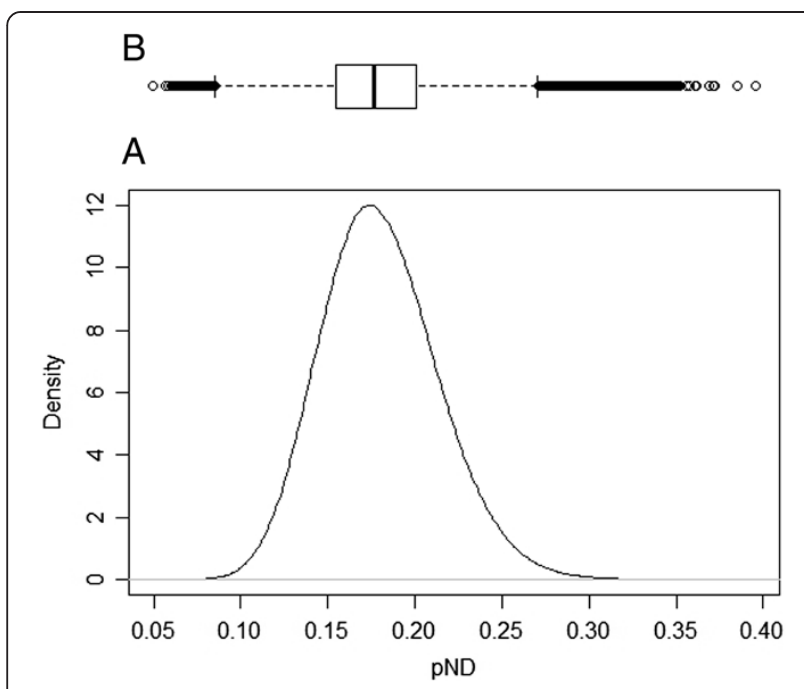

Figure 6 Posterior distribution of the probability of non-detection of infected animals ( $p N D)$. Legend: A) Plot of the probability of non-detection of infected animals ( $p N D)$ due to the test characteristics; B) Boxplot of the posterior samples used to produce the plot where the thick line in the box reflects the median.

it is important to take into consideration that the time interval used in this study was two weeks whereas in past simulation studies it varies from one day $[14,17]$ to one week [18]. As the transmission parameters are rates, however, they can be easily transformed to relate to different time steps. Although the herds used in the Kranker study [10] had moderate to high levels of Salmonella Typhimurium prevalence, the median estimates of the transmission parameters from this study are lower than those found in previous simulation studies $[16,18]$. The transition rate $\beta$ from $S$ to I is slightly higher when compared to the Lurette et al. study [18] although the other rates $(\alpha, v)$ are lower than the equivalent parameters in that same study. So the application of our proposed modelling framework to these Danish herds resulted in estimates comparable to similar studies (note that this approach could easily be used with data from other countries). Moreover, the prevalence of Salmonella in finishing pigs in Denmark is known to be close to the average prevalence in the EU [31].

To the best of our knowledge this is the first study of transmission rate parameters for Salmonella Typhimurium in swine that estimates the parameters using field data and a Bayesian probabilistic approach incorporating random effects.

\section{Bias of the study}

Correct classification of the infectious status of the individual pig is difficult for Salmonella Typhimurium infection, because the diagnostic tests currently used are imperfect [32-37]. Bacteriology lacks sensitivity given intermittent shedding of Salmonella by infected pigs, whereas using serology in individuals can be associated more with a past exposure to the agent than a current exposure; therefore it can lack specificity for detecting shedding animals. Positive serology also shows a delay between infection and expression, leading to some lack of sensitivity. When analysing the data, the lack of sensitivity was accounted for by: i) starting the analysis when at least one infected pig per cohort was observed and ii) using the probabilistic framework to predict the infectious animals that were not detected with these tests, from appropriately informed distributions based on the sensitivity of each test.

For optimal estimation of transmission parameters, the time step between each sampling should preferably be as short as the average generation interval, spanning the time when one animal becomes infectious to the time when a second animal becomes infectious because of the first animal. The time steps in this data (two weeks) are not ideal - preferably a few days or perhaps one week would be better [13]. However as previously discussed, the available data did not allow for such an option and it would be very costly to obtain new data. As data from a published study [10] were used, the time step was set to be an approximation of the different testing intervals within and between cohorts, given the limitations offered by the original set of data, and an approximation to the time of seroconversion $[13,38]$. This approximation could have affected the estimation of parameters due to the extended time interval between testing occasions. Nevertheless, comparison with the results published in other studies does not seem to support this hypothesis. Concerning the cohorts, it is clear from the Kranker study [10] that particular attention was paid to the selection of the herds, which were taken from a large population of Danish finishing herds with a well-known status for Salmonella. This gave us confidence regarding the generalization of our results, at least for herd infected with the same serotype ( $S$. Typhimurium).

\section{Transition parameters and $R_{O}$ values}

Note that the stochastic SIR model presented here is only a discrete-time approximation to the real transmission dynamics, i.e. limited to bi-weekly intervals. In particular, when the number of susceptible animals is small and the infection intensity high, then the expected number of infectious animals will tend to be overestimated [5].

The estimate of the transition rate $\beta$ (from $S$ to $\mathrm{I}$ ) is low compared with other infectious diseases (such as swine influenza) and reflects the fact that in most of the herds, Salmonella does not cause outbreaks but maintains a residual level of infection represented by infectious and carrier animals that enable the infection to 
persist in the herds. The credible interval for the transition parameter $\alpha$ (from I to R), matches the variability of shedding duration that is known from experimental and field studies $[13,39]$. The transition rate estimate $v$ (from $\mathrm{R}$ to $\mathrm{I}$ ) is small and possibly dependent on stress events (the authors of the Kranker et al. study [10] report that two cohorts in which animals began shedding in a second round had a slurry overflow which can be considered a stressful event). The variance of the cohort time-dependent random effect was high and a possible explanation for that is the different management of cohorts which in turn induces high variability (between cohorts) in the transmission data. In future studies this should be taken into consideration as a way to minimize transmission of infection.

For spread to occur, $R_{O}$ should be above one. Looking at Figure 5 we can see that there is high probability that $R_{O}>1$, 94\% specifically. The median $R_{O}$ value was 1.91 indicating that Salmonella Typhimurium was spreading in most of the cohorts. The value is not high (third quartile of $R_{O}$ is less than three) implying it would not spread rapidly through the susceptible populations under management systems similar to the ones used in these herds. With lower probability, $R_{O}$ is high enough to cause outbreaks, e.g. probability that $R_{O}>5$ is $2.5 \%$.

The $R_{O} 95 \%$ Credible Interval $(\mathrm{CrI})$ ranges from 0.78 to 5.24 . The higher values reflect that animals infected with a high infectious dose have a longer shedding period [13] than the ones infected with low infectious dose, and so the former can cause an outbreak. This makes sense because Salmonella Typhimurium is an agent that primarily spreads via the faecal-oral route.

Few studies are available to aid in defining infectious animals, but the experimental and field studies conducted by some authors $[13,15]$ support the duration of infectiousness used in our study.

A next step in our investigation will be to include the estimated transmission parameters $(\beta, \alpha, v)$ in a stochastic simulation model developed by the authors to simulate the spreading of Salmonella Typhimurium in swine herds and thus test the effectiveness of different control strategies.

\section{Conclusions}

A Bayesian framework was proposed, to estimate Samonella Typhimurium transmission parameters, and has been successfully implemented using data from Danish pig herds. The model extends the current established methodology of utilising GLMs to implement stochastic SIR models. Random effects were added to i) explicitly allow for cohort heterogeneity in the data (i.e. allow for the fact that pigs were grouped in cohorts), ii) capture possible unobserved cohort effects and iii) avoid the problem of overdispersion. Results in terms of posterior samples allow for direct probabilistic statements about model parameters, which may be also used in other analyses such as simulation models for testing management strategies.

The issue of underestimating infectious pigs due to testing sensitivity was addressed by predicting the number of non-detected pigs, using i) prior information about test sensitivity and ii) the observed data. In doing that, the probability of non-detection was treated as an unknown parameter which was estimated at the same time as the transmission parameters.

All model unknowns (transmission parameters, cohort random effects, non-detected pigs, probability of nondetection) were estimated simultaneously, implying that all possible sources of uncertainty were modelled, in turn giving more confidence about the estimates of the transmission parameters.

\section{Competing interests}

The authors declare that they have no competing interests.

\section{Authors' contributions}

CCG and TE were involved in the design and performed the statistical modelling analysis and drafted the manuscript. TB was involved in the design of the statistical analysis and the revision of the manuscript for intellectual content. LA and PB were involved in the revision of the manuscript for intellectual content. JNR was involved in the drafting and revision of the manuscript for intellectual content. All authors approved the final manuscript.

\section{Acknowledgements}

We would like to thank the Danish Agriculture \& Food Council and the authors of the study (Kranker et al., [10]) for allowing us to be using their data. This research was supported by FCT (PhD scholarship SFRH/BD/40932/2007 and pluriannual support of LIAAD-INESC Porto L.A). We are very grateful to Madeleine Henry for proof-reading this paper.

\section{Author details}

${ }^{1}$ ICBAS-UP - Department of Population Studies, Instituto de Ciências Biomédicas Abel Salazar - Universidade do Porto, Rua de Jorge Viterbo Ferreira, $n^{\circ} 228$, 4050-313 Porto, Portugal. ${ }^{2}$ CEMPS, University of Exeter, Harrison Building, North Park Road, Exeter, EX4 4QF, UK. ${ }^{3}$ LIAAD - INESC Porto L.A. Universidade do Porto, Rua de Ceuta, 118, 6, 4050-190 Porto, Portugal. ${ }^{4}$ FEP-UP - Faculty of Economics Universidade do Porto, Rua Dr. Roberto Frias, 4200-464 Porto, Portugal. ${ }^{5}$ DMA Danish Agriculture and Food Council, Axelborg, Axeltorv 3, DK-1609, Copenhagen, Denmark. ${ }^{6}$ ISPUP - Institute of Public Health - Universidade do Porto, Rua das Taipas, 4099-003 Porto, Portugal. " ${ }^{7}$ ERU-SRUC - Epidemiology Research Unit, Future Farming Systems, SRUC, Drummondhill, Inverness, IV2 4JZ, UK.

Received: 29 October 2013 Accepted: 16 April 2014 Published: 28 April 2014

\section{References}

1. EFSA: The community summary report on trends and sources of Zoonoses and Zoonotic Agents and Food-Borne Outbreaks in the European Union 2008. EFSA J 2010, 1496:288

2. Hurd HS, Enoe C, Sorensen L, Wachman H, Corns SM, Bryden KM, Grenier M: Risk-based analysis of the Danish pork Salmonella program: past and future. Risk Anal 2008, 28(2):341-351.

3. FCC Consortium: Analysis of the costs and benefits of setting a target for the reduction of Salmonella in slaughter pigs for European Commision Health and Consumers Directorate-General SANCO/2008/E2/036 Final Report. 2010:1-198. http://ec.europa.eu/food/food/biosafety/salmonella/ docs/fattening_pigs_analysis_costs.pdf.

4. Diekmann O, Heesterbeek JAP: Mathematical Epidemiology of Infectious Diseases: Model Building, Analysis and Interpretation. Chichester: John Wiley \& Sons Ltd; 2000. Chapter 2. 
5. Nielsen $L R$, van den Borne $B$, van Schaik G: Salmonella Dublin infection in young dairy calves: transmission parameters estimated from field data and an SIR-model. Prev Vet Med 2007, 79(1):46-58.

6. Tsutsui T, Kobayashi S, Hayama Y, Nishiguchi A, Kameyama K, Konishi M, Murakami K: Estimation of the within-herd transmission parameter of bovine leukemia virus. Prev Vet Med 2010, 95(1-2):158-162

7. Bouma A, de Jong MCM, Kimman TG: Transmission of pseudorabies virus within pig populations is independent of the size of the population. Prev Vet Med 1995, 23(3-4):163-172.

8. Yoshida N, Hara T: Global stability of a delayed SIR epidemic model with density dependent birth and death rates. J Comput Appl Math 2007 201(2):339-347.

9. Thomas E, Bouma A, Klinkenberg D: A comparison of transmission characteristics of Salmonella enterica serovar Enteritidis between pair-housed and group-housed laying hens. Vet Res 2011, 42:40. http:// www.veterinaryresearch.org/content/42/1/40

10. Kranker S, Alban L, Boes J, Dahl J: Longitudinal study of Salmonella enterica Serotype Typhimurium infection in three Danish farrow-to-finish swine herds. J Clin Microbiol 2003, 41(6):2282-2288.

11. Harris IT: Serologic basis for assessment of subdinical Salmonella infection in swine: part 1. J Swine Health Prod 2003, 11(5):247-251.

12. Eriksson $E$, Aspan A: Comparison of culture, ELISA and PCR techniques for salmonella detection in faecal samples for cattle, pig and poultry. BMC Vet Res 2007, 3:21

13. Fravalo P, Cariolet R, Proux K, Salvat G: Le portage asymptomatique de Salmonella enterica par les porcs: résultats issues de la constituition d'un modèle en conditions expérimentales. In 35èmes Journées de la Recherche Porcine: 2003. Paris, France: ITP, INRA; 2003:393-400.

14. Soumpasis I, Butler F: Development and application of a stochastic epidemic model for the transmission of Salmonella Typhimurium at the farm level of the pork production chain. Risk Anal 2009, 29(11):1521-1533.

15. Nielsen B, Baggesen D, Bager F, Haugegaard J, Lind P: The serological response to Salmonella serovars typhimurium and infantis in experimentally infected pigs. The time course followed with an indirect anti-LPS ELISA and bacteriological examinations. Vet Microbio/ 1995, 47(3-4):205-218.

16. Hill AA, Snary EL, Arnold ME, Alban L, Cook AJ: Dynamics of Salmonella transmission on a British pig grower-finisher farm: a stochastic model. Epidemiol Infect 2008, 136(3):320-333.

17. van der Gaag MA, Vos F, Saatkamp HW, van Boven M, van Beek P, Huirne RBM: A state-transition simulation model for the spread of Salmonella in the pork supply chain. Eur J Oper Res 2004, 156(3):782-798.

18. Lurette A, Belloc C, Touzeau S, Hoch T, Ezanno P, Seegers H, Fourichon C: Modelling Salmonella spread within a farrow-to-finish pig herd. Vet Res 2008, 39(5):49

19. Velthuis AGJ, De Jong MCM, Kamp EM, Stockhofe N, Verheijden JHM: Design and analysis of an Actinobacillus pleuropneumoniae transmission experiment. Prev Vet Med 2003, 60:53-58.

20. Broens EM, Espinosa-Gongora C, Graat EAM, Vendrig N, Van der Wolf PJ, Guardabassi L, Butaye P, Nielsen JP, De Jong MCM, Van De Giessen AW: Longitudinal study on transmission of MRSA CC398 within pig herds. BMC Vet Res 2012, 8(58):10.

21. Romagosa A, Allerson M, Gramer M, Soo Joo H, Deen J, Detmer S, Torremorell M: Vaccination of influenza a virus decreases transmission rates in pigs. BMC Vet Res 2011, 42(120):1-15.

22. Geenen PL, Dopfer D, Van der Meulen J, De Jong MC: Transmission of F4+ E. coli in groups of early weaned piglets. Epidemiol Infect 2005, 133(3):459-468.

23. Eble P, de Koeijer A, Bouma A, Stegeman A, Dekker A: Quantification of within- and between-pen transmission of foot-and-mouth disease virus in pigs. Vet Res 2006, 37(5):647-654.

24. Gilks WR, Richardson S, Spiegelhalter DJ: Markov chain Monte Carlo in practice. 1st edition. Boca Raton: Chapman \& Hall/CRC; 1996

25. Lunn DJ, Thomas A, Best N, Spiegelhalter D: WinBUGS - a Bayesian modelling framework: concepts, structure, and extensibility. Stat Comput 2000, 10:325-337.

26. Spiegelhalter D, Thomas A, Best N, Lunn D: WinBUGS Version 1.4.3 User Manual. In Medical Research Council Biostatistics Unit. Cambridge: MRC; 2007.

27. Best NG, Cowles MK, Vines SK: CODA Manual Version 0.30. Cambridge, UK: MRC Biostatistics Unit; 1995

28. Gelman A, Rubin DB: Inference from iterative simulation using multiple sequences. Stat Sci 1992, 7:457-511.
29. Kostoulas P, Leontides L, Enøe C, Billinis C, Florou M, Sofia M: Bayesian estimation of sensitivity and specificity of serum ELISA and faecal culture for diagnosis of paratuberculosis in Greek dairy sheep and goats. Prev Vet Med 2006, 76(1-2):56-73.

30. Congdon PD: Applied Bayesian Hierarchical Methods. London: Chapman and Hall/ CRC; 2010

31. EFSA: Report of the task force on Zoonoses data collection on the analysis of the baseline survey on the prevalence of Salmonella in slaughter pigs, PartA. EFSA J 2008, 135:111.

32. van Winsen RL, van Nes A, Keuzenkamp D, Urlings HAP, Lipman LJA, Biesterveld S, Snijders JMA, Verheijden JHM, van Knapen F: Monitoring of transmission of Salmonella enterica serovars in pigs using bacteriological and serological detection methods. Vet Microbiol 2001, 80(3):267-274.

33. Nollet N, Maes D, Duchateau L, Hautekiet V, Houf K, Van Hoof J, De Zuttera L, De Kruif A, Geers R: Discrepancies between the isolation of Salmonella from mesenteric lymph nodes and the results of serological screening in slaughter pigs. Vet Res 2005, 36(4):545-555.

34. Christensen J, Baggesen DL, Soerensen V, Svensmark B: Salmonella level of Danish swine herds based on serological examination of meat-juice samples and Salmonella occurrence measured by bacteriological follow-up. Prev Vet Med 1999, 40(3-4):277-292.

35. Farzan A, Friendship RM, Dewey CE: Evaluation of enzyme-linked immunosorbent assay (ELISA) tests and culture for determining Salmonella status of a pig herd. Epidemiol Infect 2007, 135(2):238-244.

36. Proux K, Houdayer C, Humbert F, Cariolet R, Rose V, Eveno E, Madec F: Development of a complete ELISA using Salmonella lipopolysaccharides of various serogroups allowing to detect all infected pigs. Vet Res 2000, 31(5):481-490.

37. Szabo I, Scherer K, Roesler U, Appel B, Nockler K, Hensel A: Comparative examination and validation of ELISA test systems for Salmonella typhimurium diagnosis of slaughtering pigs. Int J Food Microbiol 2008, 124(1):65-69.

38. Beloeil PA, Chauvin C, Proux K, Rose N, Queguiner S, Eveno E, Houdayer C, Rose V, Fravalo P, Madec F: Longitudinal serological responses to Salmonella enterica of growing pigs in a subclinically infected herd. Prev Vet Med 2003, 60(3):207-226.

39. Nicholson FA, Groves SJ, Chambers BJ: Pathogen survival during livestock manure storage and following land application. Bioresour Technol 2005, 96(2):135-143.

doi:10.1186/1746-6148-10-101

Cite this article as: Correia-Gomes et al:: Transmission parameters estimated for Salmonella typhimurium in swine using susceptibleinfectious-resistant models and a Bayesian approach. BMC Veterinary Research 2014 10:101.

\section{Submit your next manuscript to BioMed Central and take full advantage of:}

- Convenient online submission

- Thorough peer review

- No space constraints or color figure charges

- Immediate publication on acceptance

- Inclusion in PubMed, CAS, Scopus and Google Scholar

- Research which is freely available for redistribution 